TENDENCIAS

Revista de la Facultad de Ciencias

Económicas y Administrativas.

Universidad de Nariño

ISSN-E 2539-0554

Vol. XXI No. 2 - 2do Semestre 2020

Julio-Diciembre - Páginas 266-282

\title{
NOCIONES DEL ESCEPTICISMO PROFESIONAL DEL CONTADOR PÚBLICO EN EJERCICIO DE LAS AUDITORÍAS: UNA PERSPECTIVA TEÓRICA
}

\section{NOTIONS OF THE PROFESSIONAL SKEPTICISM OF THE PUBLIC ACCOUNTANT IN EXERCISE OF THE AUDITS: A THEORETICAL PERSPECTIVE}

\section{NOÇÕES DE CEPTICISMO PROFISSIONAL DO CPA NO EXERCÍCIO DAS AUDITORIAS: UMA PERSPECTIVA TEÓRICA}

\author{
ESPINOSA DÍAZ_Yuli Samary; REVELO MANTILLA_Jessica Tatiana; \\ BALLESTEROS SARABIA_ Victor Manuel
}

Magíster (C) en Gestión y Evaluación de Proyectos de Inversión, Universidad Externado de Colombia. Docente Facultad de Contaduría Pública, Universidad Santo Tomás - seccional Bucaramanga. E-mail: yuli.espinosa@ustabuca.edu.co, Colombia.

Estudiante de Contaduría Pública, Universidad Santo Tomás, Seccional Bucaramanga, E-mail: jtrm2706@gmail.com, Colombia.

Estudiante de Contaduría Pública, Universidad Santo Tomás, Seccional Bucaramanga, E-mail: victormanuelballesteros@ hotmail.com, Colombia.

Recibido: 5 de junio de 2020

Aprobado: 8 de septiembre de 2020

DOI: https://doi.org/10.22267/rtend.202102.150

\section{RESUMEN}

El Escepticismo es una doctrina filosófica que en el ejercicio laboral contribuye a los profesionales de Contaduría Pública a adquirir un enfoque basado en la duda sobre la veracidad y consistencia de la información que deben revisar durante la realización de una auditoría. Dudas que lo lleven a corroborar cada soporte encontrado en el proceso y que le permitan tener las bases suficientes para emitir dictámenes financieros confiables. Este trabajo expone una revisión literaria que explora los 
elementos que influyen en el escepticismo profesional en los contadores públicos que ejercen como auditores. Esta investigación se realizó mediante un enfoque cualitativo de tipo descriptivo y exploratorio basado en literatura existente. En este trabajo se halló que la independencia, el sesgo personal y los conflictos de intereses son factores claves que mejoran o desmejoran el escepticismo profesional en la ejecución del encargo de auditoría.

Palabras clave: auditoría contable; escepticismo profesional; independencia profesional; código de ética IFAC; sesgo personal.

JEL: M40; M41; M42; D11; D12; H83

\begin{abstract}
Skepticism is a philosophical doctrine that in professional practice contributes to Public Accounting professionals to acquire an approach based on doubt about the veracity and consistency of the information they should review during the performance of an audit. Doubts that lead him to corroborate each support found in the process and that allow him to have sufficient bases to issue reliable financial opinions. This work presents a literary review that explores the elements that influence professional skepticism in public accountants who act as auditors. This research was carried out using a descriptive and exploratory qualitative approach based on existing literature. In this study, it was found that independence, personal bias and conflicts of interest are key factors that improve or impair professional skepticism in the execution of the audit engagement.
\end{abstract}

Keywords: accounting audit; professional scepticism; professional independence; IFAC code of ethics; personal bias.

JEL: M40; M41; M42; D11; D12; H83

\title{
RESUMO
}

O ceticismo é uma doutrina filosófica que, na prática profissional, contribui para que os profissionais de contabilidade pública adquiram uma abordagem baseada em dúvidas sobre a veracidade e consistência das informações que eles devem revisar durante a realização de uma auditoría. Dúvidas que o levam a corroborar cada apoio encontrado no processo e que lhe permitem ter bases suficientes para emitir pareceres financeiros confiáveis. Este trabalho apresenta uma revisão literária que explora 
os elementos que influenciam o ceticismo profissional em contadores públicos que atuam como auditores. A pesquisa foi realizada com abordagem qualitativa descritiva e exploratória, com base na literatura existente. Neste estudo, verificou-se que independência, viés pessoal e conflitos de interesse são fatores-chave que melhoram ou prejudicam o ceticismo profissional na execução do trabalho de auditoría.

Palavras-chave: auditoria contabilística; cepticismo profissional; independência profissional; código de ética da IFAC; parcialidade pessoal.

JEL: M40; M41; M42; D11; D12; H83

\section{INTRODUCCIÓN}

En la actualidad existe una gran cantidad de normas que orientan y facilitan los procesos de auditoría para profesionales que se dedican a la realización de estas, de manera que los auditores puedan realizar un trabajo eficiente y veraz. El escepticismo profesional cumple un papel fundamental en el rol del auditor. En los últimos tiempos han existido problemas relacionados con corrupción empresarial, cartelización de las industrias y favoritismos en negociaciones comerciales. Así mismo el soborno, la extorsión, la recompensa por no reportar datos verídicos, preferencias a familiares y amigos, el uso abusivo del dinero para fines particulares, ha desencadenado una serie de incertidumbres económicas en la sociedad, pérdida de la confianza empresarial, especialmente en las firmas de auditoría quienes son los entes que controlan y verifican la severidad de los reportes financieros; práctica que ha sido recurrente en los últimos tiempos a nivel mundial.

No obstante, a medida que se obtiene un mayor grado de exigencia en la calidad de la auditoría, aumenta la probabilidad que un auditor encuentre más hallazgos y a su vez emita un informe evidenciando las inconsistencias tales como alteración u omisión de hechos económicos, fraudes o errores contables que repercuten en los reportes financieros de la entidad (DeAngelo, 1981: 187).

Pero el aumento en la calidad de la auditoría trae con consigo otros factores que afectan a esta durante su realización, entre estos factores se encuentran: la experiencia laboral, la independencia, la objetividad, la integridad y la competencia que influyen en la calidad del resultado de una auditoría (Sukriah y Inapty, 2009: 7). 
El término escepticismo profesional significa una actitud crítica mostrada por el auditor al evaluar las evidencias de una auditoría. Un auditor que tiene una actitud escéptica, no solo aceptará la explicación del cliente, sino que hará preguntas para obtener la razón, evidencia y confirmación del objeto examinado (Andreas, Zarefar y Rasuli, 2016: 3813). Lo anterior, permite realizar una reflexión a través de los diferentes autores que han escrito al respecto y han profundizado en el tema, indicando una disminución del riesgo en los informes de auditoría cuando el profesional tiene una actitud escéptica.

La aplicación del escepticismo profesional mejora la efectividad del procedimiento en una auditoría y reduce la posibilidad de seleccionar un procedimiento de auditoría incorrecto. Por esto, los auditores con niveles más altos de escepticismo profesional se comportan de manera sistemática (Hurtt, Eining y Plumlee, 2008: 5).

Este artículo realiza una revisión literaria sobre los factores que inciden en el fortalecimiento del escepticismo profesional de los Contadores Públicos en ejercicio de las auditorías, en su rol como auditores. Se muestran los conceptos que diferentes autores tienen respecto, los factores que influyen en el escepticismo profesional en el ejercicio de la auditoría y se plantean posibles escenarios que permiten mejorar la confiabilidad de las mismas.

\section{REFERENTES TEÓRICOS}

Para que la economía de un territorio sea estable, la información financiera de las entidades obligadas a llevar contabilidad bajo la regulación internacional y local, son certificados por Contadores Públicos y/o Revisores Fiscales según el grado de obligatoriedad que indique la norma, el cual debe ser creíble y de alta calidad, en todos los sectores económicos, tanto en entidades oficiales como en entidades privadas del país. Por lo tanto, es probable que al lograr una auditoría de calidad se genere a los stakeholders una confianza en la opinión del auditor sobre los estados financieros, con base en evidencia suficiente y adecuada obtenida por un equipo de trabajo, además de contar con valores éticos y actitudes apropiadas; con experiencia y tiempo suficiente para realizar el trabajo de auditoría. 
Existen dos fuerzas principales que motivan a los auditores a ofrecer calidad: los problemas jurídicos que pudieran tener en un futuro y su reputación. Esto se debe a que un auditor da fe y firma bajo juramento los estados financieros de las organizaciones para las que presta sus servicios e informa las situaciones que no corresponden a la realidad, de no hacerlo así estaría incurriendo en un delito, afectando su reputación y sus posibles futuros contratos. No obstante, los clientes pasan a otros auditores cuando la reputación de calidad de sus auditores actuales decrece (Skinner y Srinivasan, 2012: 1744). Existen varios factores que pueden mejorar la calidad de los resultados de la auditoría, entre estos se encuentra la independencia, la objetividad, la experiencia, la integridad y por su puesto el escepticismo profesional.

La independencia del auditor se caracteriza por su integridad ética y su enfoque objetivo durante el proceso de auditoría. La objetividad es una actitud que permite que los auditores crean en el trabajo que están realizando y no comprometan su calidad. La experiencia de los auditores permite que las revisiones de los estados financieros puedan realizarse durante tiempos específicos y con tareas determinadas (Andreas et al., 2016: 3811).

La independencia del auditor coadyuva a reforzar sus competencias de manera que logré ser más objetivo y escéptico para realizar un trabajo con mayor efectividad. La independencia es un factor principal para todos aquellos profesionales que ejercen su profesión como auditores en consecuencia existe un acuerdo general, en donde se indica que, sin independencia, la auditoría simplemente no tendría sentido (Power, 1999: 93).

Una actitud de independencia del auditor es un antecedente muy necesario de escepticismo profesional, dado que mejora la capacidad del auditor para llevar a cabo la auditoría con integridad y objetivamente, la independencia del auditor es una condición previa necesaria para los juicios y acciones del escepticismo profesional que son esenciales para la auditoría y los resultados de esta.

Hay dos dimensiones para el análisis de la independencia del auditor (Guénin-Paracini, Malsch, y Tremblay, 2015: 205). La primera dimensión es la independencia organizacional, que se relaciona con la disposición de los auditores para actuar de acuerdo con los estándares profesionales y para informar los errores encontrados durante la auditoría. La segunda dimensión de la independencia del auditor es 
la independencia operativa que está relacionada con la capacidad de los auditores para trabajar de manera diligente y efectiva para detectar anomalías, independientemente del comportamiento del auditado. La realidad de la independencia del auditor sigue siendo incierta y necesita ser constantemente negociada y renegociada en el campo (Guénin-Paracini et al., 2015: 221).

La integridad de los auditores implica no solo honestidad sino también trato justo y veraz. La integridad es un requisito previo para todos aquellos que trabajan bajo un interés público. Es esencial que los auditores actúen, con integridad, bajo criterios de honestidad, justicia, franqueza, coraje y confidencialidad.

Los auditores son percibidos como individuos interesados en el negocio de proporcionar servicios de auditoría, lo que podría indicar que los auditores son agentes oportunistas dispuestos a maximizar su utilidad, debido a que la delgada línea entre realizar una auditoría bajo un enfoque de rigurosidad total podría decantar en una disminución de sus futuros trabajos pues sus clientes podrían llegar a considerar su labor como inflexible y poco conveniente para sus organizaciones (Walters y Dangol, 2006: 108).

Por consiguiente, la calidad de la auditoría se puede afectar por los incentivos que obtengan de las organizaciones, lo cual conlleva a que auditor sesgue su opinión sobre la información financiera por conveniencias monetarias. Por ende, la situación planteada representa una amenaza de auditoría que deteriora el escepticismo profesional y a su vez el juicio profesional.

Se observa que actualmente, los clientes renuevan los servicios de auditoría dependiendo del informe final emitido, por lo cual el auditor concentra sus opiniones con la finalidad de preservar el cliente, sin tener en cuenta las consecuencias sancionatorias que puede generar los entes reguladores del Estado, pasando por alto las buenas prácticas de auditoría, coartando su escepticismo profesional (Knechel, Niemi y Zerni, 2013: 377). Algunos auditores no están dispuestos a confrontar a sus clientes por el temor de la no continuidad del encargo de auditoría, a pesar que estas situaciones vayan en desacuerdo con las virtudes de la profesión y su deber con el interés público (Reynolds y Francis, 2001: 381). 
Por otra parte, Gay (2002: 66) indica que los clientes influyen en los objetivos y estrategias del servicio profesional de auditoría, sin importar que tanto auditores como clientes tienen como prioridad servir al interés general con resultados creíbles y fiables de las auditorías planeadas y ejecutadas; en ciertas ocasiones se prioriza los intereses particulares sobre el interés general.

La estructura auditor-cliente expone las relaciones de poder entre las partes debido a los mayores poderes de negociación de los clientes para influir en el precio de una auditoría (Guénin-Paracini et al., 2015: 225). Por tanto, los auditores con el tiempo pueden ser potencialmente menos independientes en su función con sus clientes, derivado de los incentivos y beneficios que puedan lograr éstos en sus encargos, inclinando así la balanza a una mayor dependencia de parte del profesional ante sus clientes y acomodando las auditorías a conveniencia de las partes.

Otro factor importante que afecta la calidad de la auditoría es la confianza. Los niveles básicos de confianza funcionan como un requisito previo para las empresas y facilitan la interacción y el orden social (Bachmann, Knights y Sydow, 2001: 338). Los auditores deben extender un elemento de confianza hacia los directivos de las empresas para las que prestan sus servicios y enfocar sus resultados en los estados financieros. Los auditores otorgan un alto grado de importancia al confiar en sus clientes (Rennie, Kopp y Lemon, 2010: 290); sin embargo, esta confianza generada en su relación con el cliente no debe generar un vínculo y un acercamiento que debilite la independencia mental y el grado de escepticismo profesional para valorar las pruebas y aplicar nuevos procedimientos que conlleven con la veracidad de la información.

En la evolución del escepticismo profesional las primeras auditorías implicaron una confianza explícita en altos mandos de las empresas y se basaron significativamente en la información entregada por el personal del cliente (Matthews, 2006: 112), en la década de 1930, los auditores atenuaron cada vez más su confianza en la administración (Byrnes, Al-Awadhi, Gullvist, Brown-Liburd, Teeter, Warren y Vasarhelyi, 2018: 289).

Inicialmente se deseaba que los auditores mantuvieran una posición neutral ante sus clientes; no se esperaba que buscaran específicamente el fraude, pero debían estar alertas ante esta posibilidad. Recientemente, se ha requerido que los auditores diseñen sus planes de trabajo con el fin de 
proporcionar una seguridad razonable sobre los estados financieros mediante la detección de fraudes materiales (Bell, Peecher y Solomon, 2005: 66). A diferencia de la adopción de una posición neutral, la duda sobre la información que se está evaluando en una auditoría, supone un grado de deshonestidad hasta que la evidencia sugiera lo contrario (Bell et al., 2005: 66). Se espera que los auditores implementen procedimientos y herramientas más rigurosas desde el momento de la planeación, realizando nuevas pruebas sobre los procesos que auditan en las compañías a las que prestan sus servicios.

La confianza y la desconfianza de un auditor impactan el uso adecuado del escepticismo profesional en su comportamiento y juico profesional debido a que este último, está relacionado con la confianza que presenta el auditor con referencia al cliente y la información que este le brinda en el encargo de auditoría (McKnight y Chervany, 2001: 31). Por tanto, la expresión de confianza reduce la medida en que los auditores cuestionan y someten la evidencia a una evaluación crítica, llevando con ello a una disminución de los seguimientos y aplicación de nuevas pruebas y procedimientos.

Los niveles altos y bajos de desconfianza están representados por variaciones en el miedo, el cinismo, la cautela, la vigilancia y el escepticismo (Lewicki, McAllister y Bies, 1998: 440). Las investigaciones realizadas por otros autores demuestran que los auditores con niveles más bajos de confianza hacen juicios más escépticos (Quadackers, Groot y Wright, 2014: 643).

No obstante, el auditor requiere que en su actuar prevalezca la duda presunta sobre los hechos económicos y la información entregada por el cliente, de manera que recurra a aplicar nuevas herramientas e instrumentos que permitan detectar la veracidad de los reportes financieros.

Los niveles de desconfianza que se experimentan son un factor diferenciador de los individuos en términos del nivel de escepticismo que se aplica en el entorno (Lewicki et al., 1998: 451). Se ha demostrado que aquellos profesionales que exhiben mayores niveles de desconfianza emplean más estrategias de procesamiento no rutinarias (Schul, Mayo y Burnstein, 2008: 1298) con el fin de tener mayor certeza sobre la veracidad de la documentación que están auditando y lograr emitir un informe de auditoría con mayor confianza para las partes interesadas. 
Los auditores con mayor nivel de desconfianza exhiben una mayor creatividad y flexibilidad cognitiva (Mayer y Mussweiler, 2011: 1269). Por consiguiente, los auditores deben ser más conscientes de la responsabilidad y los riesgos que asumirían en su rol profesional al emitir un juicio que no corresponda a la realidad de la información financiera que evalúan y las posibles implicaciones legales que esto conllevaría.

La confianza es necesaria para que una auditoría sea factible (Richard, 2006: 161) pero demasiada confianza puede debilitar la independencia, lo que afecta la capacidad del auditor para actuar objetivamente y mantener una actitud de escepticismo profesional.

Por otra parte, las Normas Internacionales de Auditoría - NIA - precisan que el escepticismo profesional debe mantenerse desde el momento de la planeación como en todo el desarrollo de la auditoría, pues esto permitirá identificar y evaluar los riesgos importantes de la información incorrecta, al obtener evidencia de auditoría suficiente y adecuada sobre la veracidad de la información, al formar una opinión sobre los estados financieros basados en conclusiones extraídas de la evidencia obtenida (IAASB, 2014: 19).

El escepticismo profesional es necesario para la evaluación crítica de la evidencia de auditoría, que incluye el cuestionamiento de la evidencia de la auditoría contradictoria, la confiabilidad de los documentos, las respuestas a las consultas e información adicional obtenida de los clientes y de los directivos de las organizaciones.

Al mismo tiempo, el conflicto de intereses hace referencia a la contracción que resulta entre la postura profesional que debe mantener el auditor con relación al vínculo con el cliente y los intereses de este particularmente, es decir, el conflicto de intereses para los auditores que suscita entre la elección del interés personal frente a su obligación profesional de actuar en aras del interés público (Moore, Tetlock, Tanlu y Bazerman, 2006: 15). La evidencia de la auditoría puede ser subjetiva a la perspectiva del profesional, lo cual afecta el juicio sobre la veracidad de las proposiciones de los estados financieros que se presentan para ser revisados. Este conflicto de interés puede generar un sesgo personal, el cual está relacionado con todas las amenazas de las categorías de independencia descritas en las NIAS. 
Los juicios sesgados surgen porque los auditores no pueden separar la evaluación de la evidencia del cliente por los conceptos preconcebidos del mismo, que distorsionan los hallazgos de forma subjetiva hacia sus puntos de vista (Jenkins y Haynes, 2003: 151).

La falta de independencia profesional de los auditores puede derivarse de un sesgo inconsciente, que origina la naturaleza humana como cualquier acto de voluntad (Rodgers, Guiral y Gonzalo, 2009: 351). De igual forma, se considera incluso que los auditores más profesionales y honestos tendrán un sesgo inconsciente, pero, sin embargo, real (Bazerman y Loewenstein, 2001: 28).

El sesgo inconsciente es generalizado porque las personas rara vez creen que se aplica a ellos personalmente (Moore et al., 2006: 23). Inconscientemente, los hechos se analizan críticamente, por lo que se descuentan aquellos hechos que contradicen las conclusiones, dejando solo la evidencia que respalda una posición acríticamente adoptada.

Diferentes estudios demuestran que los auditores experimentados no son inmunes a los prejuicios y tienen más probabilidades de acceder a los números contables sesgados de un cliente que generar dichos números por sí mismos (Boyd, 2004: 390). En dicho sentido, lo generalizado sobre el prejuicio personal está dado por su propia naturaleza, es decir, suele ser invisible. La revisión de una auditoría no detectaría errores atribuibles a sesgos personales. Por lo tanto, es difícil discernir si una falla de auditoría se debe a un sesgo o a un acto de corrupción. No obstante, el sesgo del auditor es real. La existencia de sesgos cognitivos y psicológicos que hacen que los auditores lleguen a decisiones marginales a favor del cliente claramente dificulta que un auditor mantenga una actitud de independencia. Tal sesgo personal tendría efectos negativos en los juicios, acciones y resultados de escepticismo profesional. Los auditores y la profesión de auditoría deben ser conscientes sobre el conflicto de intereses, lo cual puede concluir en sesgos tanto conscientes como inconscientes, que afectan el escepticismo profesional y el juicio profesional, tanto en contabilidad como en auditoría.

Los auditores deben monitorear el comportamiento ético de la virtud para garantizar que su independencia no se vea comprometida por un sesgo inconsciente del auditor (Rodgers et al., 2009: 357). En pocas palabras, implica un marco moral, en lugar de económico, por ende, los auditores deben mantener la ética profesional en su actuar, prevaleciendo el interés común que el particular. 


\section{METODOLOGÍA}

La metodología empleada para este estudio es de corte cualitativo de tipo descriptivo y exploratorio. El estudio descriptivo consiste en "especificar las propiedades, las características y los perfiles de personas, grupos, comunidades, procesos, objetos o cualquier otro fenómeno que se someta a un análisis" (Hernández, Fernández y Baptista, 2003: 80). El estudio se basó en indagar y recolectar los factores claves que influyen en el escepticismo profesional y que así mismo afectan la calidad de la auditoría; para ello se realizó una búsqueda exhaustiva en las diferentes fuentes de información y bases de datos científicas, utilizando los troncadores de búsqueda como se ilustra en la Tabla 1.

\section{Tabla 1}

Ecuaciones de búsqueda empleadas para el objeto de estudio

\begin{tabular}{lc}
\hline Ecuación de búsqueda & Resultados \\
\hline professional AND skepticism & 573 \\
professional AND skepticism, open access & 68 \\
professional AND skepticism, en ingles & 548 \\
professional AND skepticism AND audit & 97 \\
professional AND skepticism AND audit, open access & 14 \\
professional AND skepticism AND audit - (en ingles) & 96 \\
\hline
\end{tabular}

Fuente: elaboración propia.

Una vez se seleccionaron los 97 artículos que cumplían con la ecuación de búsqueda que contenía los conceptos de escepticismo, profesional y auditoría, fueron organizados y analizados, teniendo en cuenta el año de publicación dando prioridad a los publicados recientemente. Una vez realizado este trabajo, de los 97 se seleccionaron 67 como los de mayor interés y en el presente trabajo exponemos el análisis de los 29 artículos de mayor relevancia para esta investigación.

Por último, se realizó el análisis sobre los principales artículos con las ideas más importantes y los aspectos más relevantes para el objeto de estudio. 


\section{DISCUSIÓN}

El propósito de este documento es contribuir a una mejor comprensión de la importancia del escepticismo profesional en la ejecución de una auditoría por parte de los contadores públicos, resaltando los factores que más afectan al auditor como la independencia, integridad, confianza, conflicto de intereses y el sesgo personal. El análisis de la literatura existente indica claramente que la independencia del auditor, es uno de los factores principales que garantiza una auditoría de calidad de parte de los contadores públicos dedicados a ser auditores. La anterior afirmación se apoya en el código de ética del IFAC (IFAC, 2018: 27), el cual especifica que la actitud mental de independencia en un contador público le brinda la posibilidad de realizar conclusiones sin estar bajo diferentes influencias que involucren su juicio profesional, de esta manera el contador puede actuar bajo criterios objetivos, íntegros y con escepticismo profesional. La independencia permite lograr al auditor un trabajo más objetivo y veraz en las pruebas, siendo así más integro en su actuar profesional y permeando cada acción con una actitud escéptica. Sin embargo, los autores estudiados resaltaron que la independencia total del auditor es poco probable debido al interés propio del auditor y los conflictos de intereses como consecuencia de la relación con los clientes a los cuales audita.

Adicionalmente, los juicios profesionales de los auditores se ven afectados por prejuicios personales conscientes e inconscientes, pues tales amenazas a la independencia del auditor reducen el escepticismo profesional de los contadores públicos.

Los inspectores de las auditorías deben tener en cuenta que no es suficiente evaluar el escepticismo profesional para validar los resultados de la auditoría, sino que debe tener en cuenta las implicaciones como la relación del auditor y cliente que va a ser auditado, el conflicto de intereses y el sesgo personal. Los auditores deben ser conscientes de las dimensiones éticas de sus decisiones y se les debe recordar constantemente que supervisen los comportamientos éticos que garanticen que su independencia no se ve comprometida (Rodgers et al., 2009: 361).

Otro factor clave que afecta la eficiencia de los auditores en su ejercicio profesional e impacta de manera importante el escepticismo profesional es la experiencia profesional de los auditores, debido 
a que la experiencia le brinda un mayor conocimiento para planificar y revisar la información financiera que audita.

El nivel de confianza entre un auditor y su cliente puede deteriorar con el tiempo la calidad de las decisiones del auditor y en consecuencia afecta el escepticismo profesional a medida que las relaciones entre el auditor y el cliente se alarguen. Por lo consiguiente, sus decisiones en los resultados de una auditoría con respecto a la independencia solo están dictadas por el interés propio.

La relación continua de un auditor con un cliente puede conducir a una familiaridad creciente y excesiva y amenazar la independencia del auditor. A medida que se alarga la relación auditor-cliente, la confianza se fortalece, sin embargo, esta confianza no debe limitar las funciones del auditor, quebrantando el escepticismo profesional y por ende sesgue la opinión en el informe de auditoría, como consecuencia de la relación establecida entre auditor y el cliente.

Los profesionales que enfrentan conflictos de intereses pueden encontrar difícil, si no imposible, simplemente elegir la objetividad, a pesar de los mejores esfuerzos para poner los intereses públicos por encima del cliente y mantener la objetividad, la independencia sigue siendo un problema incluso para el auditor más moral y honesto.

Por tanto, puede deberse a los sesgos propios de los auditores orientados a beneficiar en primera medida el interés personal de conservar sus clientes, llegando así a favorecer con sus decisiones a las organizaciones a las que presta sus servicios en los resultados de la información que audita. Este sesgo puede ser inconsciente a la persuasión moral o la amenaza de sanciones demoradas y probabilísticas.

Lograr la independencia del auditor sería más probable el aumentar la conciencia de los auditores y el ejercicio de la auditoría sobre las dimensiones éticas de sus decisiones. La profesión contable en su conjunto y todos sus miembros deben aceptar la responsabilidad de las acciones que genera el servicio que prestan a la sociedad y carácter riguroso del concepto verídico o no que emiten sobre la información de una organización. 
La responsabilidad profesional debe ser compartida por todos los profesionales que ejercen como auditores, esto debido a que todos los profesionales tienen el deber de evaluar críticamente los posibles daños que su trabajo puede causar a la economía de una sociedad.

Este documento impulsa futuras investigaciones que podrían llevarse a cabo para examinar la validez de los factores anteriormente expuestos en esta discusión que influyen en el escepticismo profesional de los auditores, partiendo del marco moral integrado en la educación contable, lo cual puede reforzar el escepticismo del auditor y la calidad de las auditorías.

\section{CONCLUSIONES}

Los auditores requieren una formación que les permita ser más escépticos en los procedimientos y trabajos encargados a su evaluación, de manera que identifiquen los errores que conlleva el realizar una auditoría con escaso conocimiento, herramientas y evidencia insuficiente.

Otro factor clave que incide en el escepticismo profesional y por ende en el juicio profesional, es la independencia mental, pues esta condición sesga al auditor en el momento de valorar las pruebas, lo cual distorsiona la información, reflejando un dictamen poco consistente con la realidad financiera. No obstante, los auditores deben propender por un comportamiento escéptico donde no exista un sesgo personal y conflictos de intereses en la compañía auditada.

La actitud de independencia del auditor es un factor muy necesario del escepticismo profesional, el cual, fortalece la capacidad del auditor para llevar a cabo la auditoría con integridad y de manera objetiva. La independencia del auditor es una condición necesaria para los juicios y actuaciones, los cuales contribuyen de manera significativa al pensamiento crítico y escéptico del profesional, logrando así mejores resultados en los trabajos de encargo.

En síntesis, se considera que la independencia es el factor más relevante que influye en el escepticismo profesional de los contadores públicos que cumplen la labor de auditores. Se resalta la necesidad de profundizar en una investigación científica en esta área aplicando casos de estudio en Colombia, de manera que permitan ubicar el estado del escepticismo profesional en el ejercicio de auditorías. 
Para que los auditores desarrollen un deber de interés público y un compromiso para realizar auditorías de calidad, se les debe enseñar a tomar decisiones éticas equilibradas relacionadas con el deber y la virtud, que requieren no solo la habilidad técnica para saber qué hacer sino también el coraje moral para hacerlo.

Es importante que el auditor realice una auditoría bajo los lineamientos y principios éticos, aplicando las normas internacionales de auditoría, procedimientos y demás regulación vigente, manteniendo una actitud escéptica y guardando la independencia de sus acciones y decisiones frente al encargo.

\section{REFERENCIAS}

(1) Andreas, H., Zarefar, A. y Rasuli, H. M. (2016). Analysis of factors affecting the auditors'professional scepticism and audit result quality-the case of indonesian government auditors. International Journal of Applied Business and Economic Research. 14(6), 3807-3817.

(2) Bachmann, R., Knights D. y Sydow, J. (2001). Trust and Control in Organizational Relations. Organization Studies, 22(2), V-VIII.

(3) Bazerman, M. H. y Loewenstein, G. (2001). Taking the bias out of bean counting. Harvard Business Review, 79(1), 28.

(4) Bell, T. B., Peecher, M. E. y Solomon, I. (2005). The 21st Century Public Company Audit: Conceptual Elements of KPMG's Global Audit Methodology. Sweden: KPMG International.

(5) Boyd, C. (2004). The structural origins of conflicts of interest in the accounting profession. Business Ethics Quarterly, 14(3), 377-398. doi: 10.5840/beq200414325

(6) Byrnes, P. E., Al-Awadhi, A., Gullvist, B., Brown-Liburd, H., Teeter, R., Warren, J. D. y Vasarhelyi, M. (2018). Evolution of Auditing: From the Traditional Approach to the Future Audit. En Chan, D. Y., Chiu, V. y Vasarhelyi, M. A. (Ed.), Continuous Auditing (Rutgers Studies in Accounting Analytics). (pp. 285297). Emerald Publishing Limited. doi: 10.1108/978-1-78743-413-420181014

(7) DeAngelo, L. E. (1981). Auditor Size and Audit Quality. Journal of Accounting and Economics December, 3(3), 183-199. doi: 10.1016/0165-4101(81)90002-1

(8) Gay, G. (2002). Bridging the business dynamic into the audit. Australian CPA, 72(1), 66-68.

(9) Guénin-Paracini, H., Malsch, B. y Tremblay, M. (2015). On the operational reality of auditors' independence: Lessons from the field. Auditing: A Journal of Practice \& Theory, 34(2), 201-236. 
(10) Hernández, S., Fernández, C. y Baptista, P. (2003). Metodología de la Investigación. México D.F.: McGraw-Hill.

(11) Hurtt, K., Eining, M. y Plumlee, R. D. (2008). An Experimental Examination of Professional Skepticism. Doi: $10.2139 /$ ssrn. 1140267

(12) International Auditing and Assurance Standards Board-IAASB. (2014). ISA 200: Overall Objectives of the Independent Auditor and the Conduct of an Audit in Accordance with International Standards of Auditing. New York: International Federation of Accountants (IFAC).

(13) International Federation of Accountants-IFAC. (2018). Código internacional de ética para profesionales de la contabilidad (incluidas normas internacionales de independencia). Recuperado de https://www.ifac.org/system/files/publications/files/Final-Pronouncement-The-Restructured-CodeES.pdf

(14) Jenkins, J. G. y Haynes, C. M. (2003). The persuasiveness of client preferences: an investigation of the impact of preference, timing and client credibility. Auditing: A Journal of Practice and Theory, 22(1), 143-154.

(15) Knechel, W. R., Niemi, L. y Zerni, M. (2013). Empirical evidence on the implicit determinants of compensation in Big 4 audit partnerships. Journal of Accounting Research, 51(2), 349-387. doi: 10.1111/1475-679X.12009

(16) Lewicki, R. J., McAllister, D. J. y Bies, R. J. (1998). Trust and Distrust: New Relationships and Realities. The Academy of Management Review, 23(3), 438-458. doi: 10.2307/259288

(17) Matthews, D. (2006). A History of Auditing: The Changing Audit Process in Britain from the Nineteenth Century to the Present Day. New York: Routledge Taylor \& Francis Group.

(18) Mayer, J. y Mussweiler, T. (2011). Suspicious Spirits, Flexible Minds:When Distrust Enhances Creativity. Journal of Personality and Social Psychology, 101(6), 1262-1277.

(19) McKnight, D. H. y Chervany, N. L. (2001). Trust and Distrust Definitions: One Bite at a Time. En Falcone, R., Singh, M. y Tan, Y. H. (Eds), Trust in Cyber-Societies (pp. 27-54). Berlin, Heidelberg: SpringerVerlag. doi: 10.1007/3-540-45547-7_3

(20) Moore, D. A., Tetlock, P. E., Tanlu, L. y Bazerman, M. H. (2006). Conflicts of interest and the case of auditor independence: Moral seduction and strategic issue cycling. Academy of Management Review, 31(1), 10-29. doi: 10.5465/amr.2006.19379621

(21) Power, M. (1999). The Audit Society: Rituals of verification. New York: Oxford University Press.

(22) Quadackers, L., Groot, T. y Wright, A. (2014). Auditors' Professional Skepticism: Neutrality versus Presumptive Doubt. Contemporary Accounting Research, 31(3), 639-657. doi: 10.1111/1911-3846.12052 
(23) Rennie, M. D., Kopp, L. S. y Lemon, W. M. (2010). Exploring Trust and the Auditor-Client Relationship: Factors Influencing the Auditor's Trust of a Client Representative. Auditing: A Journal of Practice and Theory, 29(1), 279-293. doi: 10.2308/aud.2010.29.1.279

(24) Reynolds, J. K. y Francis, J. (2001). Does size matter? The influence of large clients on office-level auditor reporting decisions. Journal of Accounting and Economics, 30(3), 375-400.

(25) Richard, C. (2006). Why an auditor can't be competent and independent: a French case study. European Accounting Review, 15(2), 153-179. doi: 10.1080/09638180500104832

(26) Rodgers, W., Guiral, A. y Gonzalo, J. A. (2009). Different pathways that suggest whether auditors going concern opinions are ethically based. Journal of Business Ethics, 86(3), 347-361. doi: 10.1007/s10551008-9851-8

(27) Schul, Y., Mayo, R. y Burnstein, E. (2008). The Value of Distrust. Journal of Experimental Social Psychology, 44(5), 1293-1302. doi: 10.1016/j.jesp.2008.05.003

(28) Skinner, D. J. y Srinivasan, S. (2012). Audit Quality and Auditor Reputation: Evidence from Japan. The Accounting Review, 87(5), 1737-1765. doi: 10.2308/accr-50198

(29) Sukriah, I. y Inapty, B. A. (2009). Pengaruh Pengalaman Kerja, Independensi, Obyektifitas, Integritas dan Kompetensi Terhadap Kualitas Hasil Pemeriksaan. Simposium Nasional Akuntansi, 12, 3-9.

(30) Walters, J. E. y Dangol, R. (2006). Ethical implications of independent quality auditing. Asian Journal of Information Technology, 5(1), 107-110.

Cómo citar este artículo: Espinoza, Y., Revelo, J. y Ballesteros, V. (2020). Nociones del escepticismo profesional del Contador Público en ejercicio de las auditorías: una perspectiva teórica. Tendencias, 21(2), 266282. https://doi.org/10.22267/rtend.202102.150 